\title{
Etude de la qualité physico-chimique et technologique d'une variété locale (Kitombe) et quatre variétés améliorées du riz pluvial (Lioto, Liboga, Lienge et Nerica7) cultivées à Kisangani et ses environs, en R.D.Congo
}

\author{
Liboga Oenabaiso Bienvenu1*, Litucha Bakokola Joseph1., Ngama Boloy Faustin1., Balimo Isecha \\ Fidele $^{2}$ \\ 1 Institut Facultaire des Sciences Agronomiques de Yangambi, Département de Phytotechnie (IFA-Yangambi), B.P \\ 1232 Kisangani, B.P.28 Yangambi, R.D.Congo. \\ 2 Inspection provinciale de l'agriculture, pèche et élevage (IPAPEL), B.P. 1714 Kisangani, R.D.Congo. \\ * Auteur correspondant, e-mail:bienvenuliboga@yahoo.fr; Téléphone: (+243)971888175
}

Original submitted in on 23rd April 2019. Published online at www.m.elewa.org/journals/ on $31^{\text {st }}$ July 2019 https://dx.doi.org/10.4314/jab.v139i1.3

\section{RÉSUMÉ}

Objectif : Le riz (Oryza sativa L.) est une denrée alimentaire très importante en R.D.Congo. A Kisangani et ses environs plusieurs variétés sont cultivées mais leurs caractéristiques physico-chimique, technologique et nutritionnelle ne sont pas connues. Ce travail s'inscrit dans cette dynamique de caractérisation physicochimique et technologues de quelques variétés les plus cultivées.

Méthodologie et résultats: Pour déterminer la valeur alimentaire et technologique des grains et encourager le choix de variété à préparer, les profils chimiques, minéraux et technologiques de 5 variétés les plus cultivées à Kisangani ont été étudiés. Les résultats obtenus montent que les variétés étudiées ont une composition approximative en éléments nutritifs et physico-chimiques comme celle reconnue pour le riz (Oryza sativa L.) mais varie en fonction des variétés ( $p<0,05)$. Les protéines varient de $6,2 \%$ (Nerica7) à $7,65 \%$ (Kitombe),cendres brutes de 0,8\%(Nerica7) à 1,62\%(Lienge), $\mathrm{Mg}$ de $0,39 \%$ (Lienge) à $0,71 \%$ (Kitome),pourcentage d'élongation de 35,18\%(Liboga) à 50,63\%(Kitombe), taux de brisures de $3,12 \%$ (Kitombe) à 4,59\%(Lienge)

Conclusion et application des résultats : Cette étude de la qualité physico-chimique et technologique de cinq variétés de riz pluvial a permis la découverte de plusieurs informations qui étaient jusque-là lacunaires. La variété locale dite Kitombe dont la couleur du caryopse est rouge regorge de bonnes qualités nutritionnelles, notamment la teneur élevée en protéine, en Mg et le pouvoir de gonflement à la cuisson. Ce dernier serait parmi les points forts de cette variété de riz, c'est ainsi que dans le contexte de pauvreté que sont confrontés les congolais en générale, les consommateurs de Kisangani portent leur choix à cette variété pour permettre aux familles les plus démunies de se partager les petites quantités. Bien que les différences trouvées ne soient pas statiquement différentes, les valeurs moyennes obtenues montrent que la variété Liboga est plus riche en matières sèche et en fer Lioto en lipide et Lienge en cendres brutes. La variété Nerica7 est moins riche en protéine mais de même teneur en calcium que Liboga Lienge et Kitombe. Les résultats trouvés dans ce travail constituent une base solide pour 
l'amélioration génétique du riz en permettant de faire le choix de géniteurs sur base de leurs caractéristiques physico-chimique et technologiques.

Mots clés : Riz, valeur nutritive, éléments minéraux, qualité technologique

Study of the physicochemical and technological quality of a local variety (Kitombe) and four improved varieties of upland rice (Lioto, Liboga, Lienge and Nerica7) cultivated at Kisangani and its surroundings in R. D. Congo

\section{ABSTRACT}

Objectives: Rice (Oryza sativa L.) is a very important food in D.R.Congo. At Kisangani and its surroundings, many varieties are cultivated but their physicochemical and technological characteristics are not known. This work is carried out to characterize the most cultivated varieties

Methodology and Results: to determine the nutritional and technological value of the grains and to encourage the choice of variety to prepare, the chemical, mineral and technological profile of 5 most cultivated varieties was studied. The results obtained show that the varieties studied have an approximate composition in physicochemical and nutritional elements such as recognized for rice (Oryza sativa L.) but varies according to varieties $(p<0.05)$. Proteins range from $6.2 \%$ (Nerica7) to $7.65 \%$ (Kitombe), Crude ash from $0.8 \%$ (Nerica7) to $1.62 \%$ (Lienge), $\mathrm{Mg}$ from $0.39 \%$ (Lienge) to $0.71 \%$ (Kitombe), elongation percentage $35.18 \%$ (Liboga) to $50.63 \%$ (Kitombe), broken rice from $3.12 \%$ (Kitombe) to $4.59 \%$ (Lienge)

Conclusions and application of findings : This study of the physicochemical and technological caracteristics of five varieties of upland rice led to the discovery of information that were previously incomplete. The local variety Kitombe, caryopsis color is red, is full of good nutritional qualities, including the high content of protein, $\mathrm{Mg}$ and the swelling power when cooked. The latter would be among the strong points of this variety of rice, thus the consumers of Kisangani should choose this variety to allow the most deprived families to share small quantities. Although the differences found are not statistically different, the average values obtained show that the variety Liboga is richer in dry matter and iron, Lioto in lipid and Lienge in ash. The variety Nerica7 is less rich in protein but has the same calcium content as Liboga Lienge and Kitombe. The results found in this work provide a solid foundation for genetic improvement of rice by allowing parents to be selected based on their physicochemical and technological characteristics.

Keywords : Rice, nutritional value, mineral elements, technological quality

\section{INTRODUCTION}

Le riz est l'une des céréales la plus importante au monde (Pandey et Shukla, 2015; Islam et al., 2018). II constitue une source d'énergie, de protéine et de plusieurs autres nutriments nécessaires pour l'alimentation des humains mais aussi pour celle des animaux (Kim et al., 2006). La valeur commerciale d'un riz se traduit par la qualité des grains à livrer aux consommateurs, celle-ci est définie comme un ensemble de caractéristiques répondant à des critères admis par les différents utilisateurs notamment le rendement à l'usinage, le taux de brisures, le format, la translucidité, les aptitudes culinaires (Okpala et al., 2017). La combinaison de ces caractéristiques séparées détermine son acceptabilité par les consommateurs (Laignelet et Marie,1983).C'est une expression phénotypique soumise aux différents facteurs comme le milieu mais reste surtout sous la dépendance de facteurs héréditaires (Simonelli et al., 2016).Vers les années 1990, I'Institut national pour l'étude et la recherche agronomiques (INERA) par le truchement de son programme national de recherche sur le riz (PNR Riz) a adopté une politique d'enrichissement de son germoplasme d'une part par l'acquisition des variétés exotiques grâce aux coopérations régionales, c'est le cas des variétés Nerica (Nouveau riz africain) issu de croisement interspécifique entre le riz asiatique (Oryza sativa L.) et le riz africain (O.glaberrima 
Steud.)(Tomonori et Kazuki, 2016) et de variétés IRAT. D'autre part, la stratégie consiste à mettre au point localement des nouvelles variétés par les croisements dirigés suivis des sélections, c'est le cas des variétés Lienge, Lioto, Inera7, Baibinge1, etc. Dans cette deuxième stratégie, les recherches ont été orientées soit pour la création des variétés qui s'adaptent bien au milieu avec un rendement en grains élevé à l'unité de surface, c'est le cas de variétés Baibinge1 et Lienge (Mateso et al.,2003), sur la résistance aux maladies ou encore sur la réduction de cycle végétatif, c'est le cas de la variété Lioto. Très peu d'attention a été accordée aux caractéristiques chimiques et nutritionnelles.

\section{MATERIELS ET METHODES}

Matériel végétal : Nous avons utilisé cinq variétés de riz pluvial (tableau 1). Les semences étaient obtenues
La connaissance de la composition en éléments nutritifs et chimiques est très utile car, les éléments tels que protéine, lipide, Fer, Magnésium et calcium jouent un rôle très important dans l'organisme humain (Béminga,2011) C'est dans cette optique que la présente étude a été réalisée dans le but de faire une caractérisation physicochimique, technologique et nutritionnelle de cinq variétés les plus cultivées à Kisangani et ses environs pour ainsi répondre aux suggestions de Liboga et al.(2018) qui ont recommandé une étude complémentaire à l'évaluation agro morphologique de ces variétés.

des essais au champ réalisés à Institut Facultaire des sciences agronomiques de Yangambi (IFA-Yangambi)

Tableau 1: Quelques caractéristiques de grains de variétés de riz expérimentées.

\begin{tabular}{|l|c|l|c|c|c|c|c|c|}
\hline Variété & Obtenteur & Origine gén. & $\begin{array}{c}\text { Origine } \\
\text { géo. }\end{array}$ & $\begin{array}{c}\text { couleur } \\
\mathbf{( 1 )}\end{array}$ & $\begin{array}{c}\text { Couleur } \\
\text { caryopse(2) }\end{array}$ & $\begin{array}{c}\text { arête } \\
\mathbf{( 3 )}\end{array}$ & $\begin{array}{c}\text { Pilosité } \\
\text { (4) }\end{array}$ & $\begin{array}{c}\text { Adaptation } \\
\text { écologique }\end{array}$ \\
\hline Lioto & INERA & R66/IRAT112 & RDC & pourpre & Blanc & - & + & riz pluvial \\
\hline Liboga & INERA & MutantIRAT112 & RDC & pourpre & Blanc & - & + & riz pluvial \\
\hline Lienge & INERA & OS6/IRAT13 & RDC & pourpre & Blanc & - & - & riz pluvial \\
\hline Nerica7 & ADRAO & (O.sativa / O.glaberrima) & Benin & pourpre & Blanc & - & - & riz pluvial \\
\hline Kitombe & & & RDC & Paille & Rouge & + & + & riz pluvial \\
\hline
\end{tabular}

Légende : + =présence de caractère; - = absence de caractère;(1-4)=observation visuelle basée sur le manuel du technicien (ADRAO, 2009)

Usinage de riz : Cinq cent grammes de riz paddy ont été décortiqués dans une décortiqueuse de poche à frottement (Guidettie Artioli-Verceli Ltd, Italia) d'utilisation habituelle au laboratoire rizier du centre de recherche de I'INERA-Yangambi pour les travaux de sélection et d'amélioration variétale du riz. Après usinage, les grains entiers ont été séparés des brisures par le triage manuel. Par le rapport en p.100 entre le poids de riz blanchi et le poids de riz paddy ventilé on a obtenu le rendement à l'usinage tel que procédé par Marie et Laignet (1983). Le taux de brisures a été obtenu par le rapport en p.100 entre le poids de grains brisés et le poids de riz entier blanchi.

Analyse de forme apparente des grains: La longueur (L), la largeur (I), l'épaisseur (e) et le rapport longueur sur largeur (L/l) des grains paddy et grains blanchi ont été mesurés à l'aide d'un pied à coulisse (Total digital calipter Ltd, China). La translucidité des grains a été déterminée après les avoir coupé transversalement. Le caractère crayeux ou cristallin a été noté à l'aide de l'échelle de codification de caractère (Dodelmann, 1976), donnée de 1 à 9 avec quelques classes repères: 1=grain translucide, $3=$ secteur opaque: le quart en coupe du grain, tache crayeuse importante, $6=$ secteur opaque: la moitié de la surface en coupe du grain; tache crayeuse très importante, 9: grain opaque. Etant donné que la distinction entre les classes 3 et 6 est souvent complexe de façon pratique, car la tache crayeuse peut être importante et de forme très variable au sein même d'une variété, nous avions groupé les classes 3 et 6 en une seule, notée 3 pour designer grains crayeux.

Dosage de protéine et de lipide : La détermination de teneur en protéine (en pourcentage de matière sèche) a été réalisée par la méthode Kjeldalh de détermination de l'azote total présent dans les échantillons, avec 6,25 comme coefficient de conversion. Les lipides (matières grasses) ont été déterminées après extraction au 
soxhlet avec de l'éther de pétrole à $40-60^{\circ} \mathrm{C}$ pendant 6 8 heures par gravimétrie.

Dosage des éléments minéraux: La détermination des éléments minéraux a été effectuée par la procédure d'attaque nitroperchlorique (Dubois, 1956). Puis chaque élément (Calcium, Magnésium et Fer) a été dosé par les méthodes utilisées pour les végétaux (Béminga, 2011) avec référence aux méthodes universelles (AOAC, 1995)

Comportement à la cuisson et capacité d'absorption d'eau : La préparation du riz varie d'un peuple à un autre. Dans cette étude, pour déterminer le volume globale de gonflement (VGG), $500 \mathrm{~g}$ de riz blanchi ont été cuits dans un bol. Avec une latte graduée, la hauteur finale atteinte par chaque variété après cuisson a été mesurée. Le pouvoir d'élongation de grains de riz cuit a été déterminé en mesurant de façon séparée sur un papier mouchoir la longueur moyenne de10 grains de riz cuit à l'aide d'un papier millimétré par la méthode de Okpal et al.(2017) de la manière suivante: $(\mathrm{E} \%)=\frac{\text { TMApC-TMAvC }}{\text { TMAvC }} 100$, où $\mathrm{E} \%$ est le pourcentage d'élongation; TMAvC: taille moyenne des grains avant cuisson et TMApC est la taille moyenne des grains après cuisson. La quantité d'eau absorbée a été déterminée dans le but de connaitre la capacité de chaque variété à absorber de l'eau. Pour cela, $1 \mathrm{~g}$ de riz blanchi non cuit a été trempé dans $15 \mathrm{ml}$ d'eau distillée à $30^{\circ} \mathrm{C}$ dans les boites de Pétri selon la procédure de Kim et al. (2006). Après 56heures, les grains gonflent et le poids dû à l'absorption d'eau a été déterminé puis converti en p.100 par la

\section{RESULTATS ET DISCUSSION}

Caractéristiques apparentes des grains de riz: Les caractéristiques morphologiques de grains de différentes variétés ont été étudiées et sont indiquées dans le tableau 2. La longueur $(\mathrm{L})$ des grains paddy varie de 8,30 à 10,21 mm, la largeur (I) de 2,92 à 3,09 $\mathrm{mm}$, l'épaisseur (e) 2,15 à 2,22 $\mathrm{mm}$ et le rapport longueur sur largeur (L/I) 2,83 à $3,30 \mathrm{~mm}$ Seul le rapport $\mathrm{L} / \mathrm{l}$ varie significativement en fonction des variétés. Moins de $25 \%$ de la surface des grains de toutes les variétés est occupée par la tache crayeuse et ont été cotées 1 sauf la variété Kitombe, cotée 3. Quant au riz blanchi, aucune différence significative n'a été trouvée sur la forme des grains. La longueur $(\mathrm{L})$ varie de 6,12 à $7,28 \mathrm{~mm}$, la largeur (I) de 2,53 à 2,72 $\mathrm{mm}$ et l'épaisseur (e) de 1,80 à 1,97 mm. La forme de grains est un caractère variétal mais peut être influencée par le climat. Une sècheresse au cours de remplissage de relation : $Q a(\%)=\frac{P f-P i}{P i} 100$, où $Q$ a représente la quantité d'eau absorbée (\%); Pf : poids des grains à la fin de trempage $(\mathrm{g})$ et $\mathrm{Pi}$, poids de grains avant trempage $(\mathrm{g})$

Autres analyses: Le taux d'humidité a été déterminé par gravimétrie selon la méthode décrite par AOAC (1995). Le taux de matière sèche (MS) s'est arbitrairement déduit du taux d'humidité, l'alkali digestible (AlkD),indicateur de la température de gélatinisation a été déterminé en plaçant de façon séparée ( à ne pas se toucher) dans des boites de Pétri 6 grains de riz blanchi dans $10 \mathrm{ml}$ de $\mathrm{KOH} 1,7 \%$ à $30^{\circ} \mathrm{C}$ pendant 23heures, puis avons fait la lecture suivant l'échelle du système standard d'évaluation du riz (SES) de I'Institut International de recherche sur le riz, IRRI (IRRI,2002). La teneur en cendres brutes (CB) a été obtenue après calcination des échantillons dans un four à moufle à $555^{\circ} \mathrm{C}$ (environ $5 \mathrm{~h}$ ) par la relation $\mathrm{CB}(\%)=\frac{\mathrm{P} 2}{\mathrm{P} 1} 100$, où $\mathrm{CB}$ représentent les cendres brutes(\%); P2, poids de l'échantillon après calcination(g);P1, poids de l'échantillon avant calcination(g).Les données relatives au poids ont été mesurées par une balance de précision (Radwag wigi Elektroniczne, Model : WLC 0,6/B1, Ltd, Poland).L'analyse de variance (ANOVA) avec le logiciel Minitb ${ }^{\circledR} 18$, (version française) a été utilisée pour la comparaison des moyennes. Les résultats obtenus ont été comparées entre eux et avec des valeurs moyennes reconnues dans la littérature.

grains abouti à la formation de grains de taille réduite (Liboga et al.,2018).La classification de l'union européenne (UE) de 1998 basée sur la forme des grains de riz blanchi classe les grains de riz en grains courts lorsque la longueur est inférieure à $5,2 \mathrm{~mm}$; grains moyens lorsque la longueur est inférieure à 6,0 $\mathrm{mm}$ et grains longs lorsque la longueur est de plus de 6 $\mathrm{mm}$. A l'heure actuelle, le riz à grains courts dans les marchés internationaux n'a d'importance que pour la fabrication des gâteaux ou des farines, la préférence actuelle est accordée au riz long (plus de $6 \mathrm{~mm}$ ), bien que la tendance de certains pays d'Amérique du nord est de recourir à un type de riz blanchi dit "US" dont la longueur est plus de $7 \mathrm{~mm}$ et à un type dit "Suriname" dont la longueur est supérieur à $8 \mathrm{~mm}$. Un centre crayeux (blanc, non translucide) est un défaut de qualité pour le riz (Juliano et al., 2019). 
Tableau 2: Caractéristiques physiques de grains de riz de différentes variétés étudiées

\begin{tabular}{|l|l|l|l|l|c|l|l|l|}
\hline \multirow{2}{*}{ Variétés } & \multicolumn{9}{|c|}{ riz paddy } & \multicolumn{3}{c|}{ riz blanchi } \\
\cline { 2 - 9 } & $\mathbf{L}(\mathbf{m m})$ & $\mathbf{I}(\mathbf{m m})$ & $\mathbf{e}(\mathbf{m m})$ & $\mathbf{L} / \mathbf{I}$ & Transl. (1-9) & $\mathbf{L}(\mathbf{m m})$ & $\mathbf{I}(\mathbf{m m})$ & $\mathbf{e}(\mathbf{m m})$ \\
\hline Lioto & $9,81 \mathrm{a}$ & $3,01 \mathrm{a}$ & $2,21 \mathrm{a}$ & $3,28 \mathrm{a}$ & 1 & $6,94 \mathrm{a}$ & $2,53 \mathrm{a}$ & $1,90 \mathrm{a}$ \\
\hline Liboga & $9,71 \mathrm{a}$ & $3,09 \mathrm{a}$ & $2,20 \mathrm{a}$ & $3,19 \mathrm{a}$ & 1 & $7,28 \mathrm{a}$ & $2,66 \mathrm{a}$ & $1,92 \mathrm{a}$ \\
\hline Lienge & $10,21 \mathrm{a}$ & $3,08 \mathrm{a}$ & $2,22 \mathrm{a}$ & $3,30 \mathrm{a}$ & 1 & $7,19 \mathrm{a}$ & $2,57 \mathrm{a}$ & $1,97 \mathrm{a}$ \\
\hline Nerica7 & $9,54 \mathrm{a}$ & $3,08 \mathrm{a}$ & $2,20 \mathrm{a}$ & $2,83 \mathrm{~b}$ & 1 & $6,99 \mathrm{a}$ & $2,59 \mathrm{a}$ & $1,94 \mathrm{a}$ \\
\hline Kitombe & $8,30 \mathrm{a}$ & $2,92 \mathrm{a}$ & $2,15 \mathrm{a}$ & $2,84 \mathrm{~b}$ & 3 & $6,12 \mathrm{a}$ & $2,72 \mathrm{a}$ & $1,80 \mathrm{a}$ \\
\hline MG. & 9,51 & 3,03 & 2,20 & 3,09 & & 6,90 & 2,61 & 1,90 \\
\hline Standard1 & 9,60 & 3,50 & & 2,66 & & 6,85 & 2,09 & \\
\hline S $^{2}$ (variété & 2,09 & 0,022 & 0,0025 & 0,22 & & 0,94 & 0,0072 & 0,015 \\
\hline CV $(\%)$ & 15,20 & 4,6 & 5,14 & 5,74 & & 9,85 & 20,66 & 14,91 \\
\hline LSD 0,05 & $3,16^{\mathrm{NS}}$ & $0,30^{\mathrm{Ns}}$ & $0,23^{\mathrm{Ns}}$ & $0,37^{*}$ & & $1,48^{\mathrm{NS}}$ & $1,17^{\mathrm{NS}}$ & $0,61^{\mathrm{NS}}$ \\
\hline
\end{tabular}

${ }^{*}$ Dans tous les tableaux de résultats, les valeurs suivies de mêmes lettres dans la même colonne ne sont pas significativement différentes pour $p=5 \%$; Ns: non significatif pour $p=5 \%$; Transl: translucidité; MG: moyenne générale; $S^{2}=$ variance; $\mathrm{CV}=$ coefficient de variation ; standard $1=$ valeurs moyennes obtenues à l'INERA(R.D.Congo) après expérimentation de 7 lignées pendant 2 ans (Mateso et al., 2003)

Composition générale des grains: La composition approximative de variétés de riz en différents constituants est montrée dans le tableau 3 L'analyse de la teneur en protéine montre qu'il y a variabilité en fonction de variété $(p<0,05)$. La variété Kitombe dont la couleur du riz cargo est rouge a une teneur élevée en protéine $(7,65 \%)$ alors que la plus petite teneur en protéine a été obtenue dans la variété Nerica7 $(6,2 \%)$ qui est une variété exotique. Les variétés à riz cargo rouge présentent une richesse en certains éléments (Juliano et al., 2019). Récemment, il a été démontré que l'augmentation de $\mathrm{CO}_{2}$ dans l'air, plus particulièrement au moment de l'initiation paniculaire entraine la diminution de protéine et la cristallinité des grains chez le riz (Usui et al., 2015). La qualité de riz est aussi liée à sa teneur en lipides bien que ces lipides soient de quantité très faible (Monsoor et al., 2004). Aucune différence significative n'a été trouvée entre les différentes variétés en ce qui concerne les lipides (matières grasses), mais si on se réfère à la moyenne individuelle, la variété Lioto reste supérieure aux autres variétés $(1,17 \%)$. Pour la cendre brute, seule la variété Nerica7 a montré une teneur très faible comparativement à la moyenne générale $(p<0,05)$. II s'est observée une variabilité dans la composition des différentes variétés en humidité $(p<0,05)$ avec une teneur élevée dans la variété Nerica7 $(13,9 \%)$ et une teneur faible chez la variété Liboga $(11,7 \%)$. La composition en matière sèche (MS) montre également une variabilité entre les variétés $(p<0,05)$ avec une teneur faible chez la variété Nerica7 $(86,1 \%)$. Seule la variété Liboga montre une teneur haute en Alkali digestible (AlkD), indicateur de la température de gélatinisation. L'AlkD est étroitement liée à la texture $d u$ grain chez le riz (Delwiche et al., 1996). Lorsqu'on traite les grains de riz par les bases fortes comme $\mathrm{NaOH}$ à $2 \%$ ou $\mathrm{KOH}$ à $1,7 \%$, ces grains se déforment quasiment en faisant des fentes ou vont se dissoudre complètement dans la solution en fonction de leur température de gélatinisation (Kim et al., 2006). L'amidon, composante principale de riz usiné (Kim et al., 2006) composé de amylose et amylopectine (Faur et Mazaud, 1995) n'a pas été déterminé dans notre étude faute des équipements appropriés. Mais étant donné qu'il existe déjà une corrélation positive établie entre la capacité d'absorption d'eau et la teneur en amylose mais négativement corrélée avec la faculté de coller. La variété Nerica7, aurait une moyenne en amylose supérieure aux autres variétés vu sa capacité d'absorption d'eau élevée, bien que la différence trouvée ne soit pas significativement différent $(21 \mathrm{~g}$ d'eau absorbée à 5 heures pour Nerica7 contre $12 \mathrm{~g}$ d'eau absorbée en même durée de temps pour Kitombe). Cette information sur la quantité d'eau consommée est aussi utile surtout dans la modernisation actuelle où l'on utilise de plus en plus des autocuiseurs de riz mis au point aux USA et au Japon. On corrèle également l'élasticité des grains (capacité à retrouver sa taille originelle après la première mastication) à la température de gélatinisation. Cette dernière est un bon indicateur de comportement de riz à la cuisson (Kim et al., 2006). 
Tableau 3: Composition approximative en certains constituants des différentes variétés testées,

\begin{tabular}{|l|l|l|l|l|l|l|l|l|}
\hline Variété & Hum. $(\%)$ & MS $(\%)$ & CB $(\%)$ & Protéines (\%) & Lipides(\%) & AlkD & Qa(\%) & Tgel \\
\hline Lioto & $13,3 \mathrm{bc}$ & $86,7 \mathrm{cbd}$ & $1,10 \mathrm{~b}$ & $7,22 \mathrm{ab}$ & $1,17 \mathrm{a}$ & $\mathrm{I}$ & $20,66 \mathrm{a}$ & $\mathrm{I}$ \\
\hline Liboga & $11,7 \mathrm{~d}$ & $88,3 \mathrm{a}$ & $1,10 \mathrm{~b}$ & $6,75 \mathrm{bc}$ & $0,92 \mathrm{a}$ & $\mathrm{H}$ & $19,66 \mathrm{a}$ & $\mathrm{H}$ \\
\hline Lienge & $13 \mathrm{c}$ & $87 \mathrm{~b}$ & $1,62 \mathrm{a}$ & $7,45 \mathrm{ab}$ & $0,96 \mathrm{a}$ & $\mathrm{I}$ & $18,66 \mathrm{a}$ & $\mathrm{I}$ \\
\hline Nerica7 & $13,9 \mathrm{a}$ & 86,1 & $0,80 \mathrm{~b}$ & $6,2 \mathrm{c}$ & $0,80 \mathrm{a}$ & $\mathrm{B}$ & $21,00 \mathrm{a}$ & $\mathrm{I}$ \\
\hline Kitombe & $13,4 \mathrm{~b}$ & $86,6 \mathrm{~d}$ & $1,15 \mathrm{~b}$ & $7,65 \mathrm{a}$ & $1,10 \mathrm{a}$ & $\mathrm{B}$ & $16,6 \mathrm{a}$ & $\mathrm{H}$ \\
\hline MG. & 13,1 & 86,88 & 1,14 & 7.07 & 0,97 & & 19,33 & \\
\hline Standard & 11,1 & & 0,79 & 7,27 & 1,22 & & & \\
\hline S2 $^{2}$ (variété) & 2,68 & 2,60 & 0,35 & 1,30 & 0,06 & & 9,16 & \\
\hline C.V $(\%)$ & 1,06 & 0,20 & 12,28 & 2,97 & 17,52 & & 11,07 & \\
\hline LSD 0,05 & $0,30^{*}$ & $0,39^{*}$ & $0,30^{*}$ & $0,45^{\star * *}$ & $0,37 \mathrm{NS}$ & & $4,66^{\mathrm{NS}}$ & \\
\hline
\end{tabular}

Légende: Hum: humidité. AlkD: alkali digestible. Qa: quantité d'eau absorbée. Tgel: température de gélatinisation., I: intermédiaire., H:Haute., B:basse. Ns=différence non significative pour $p=5 \% ;{ }^{* * *}$ : différence significative; pour $p=0,1 \% ;{ }^{* *}$ : différence significative pour $p=1 \% ; C B$ : cendres brutes; standard²= valeurs moyennes reconnues dans la littérature (Favier, 1989)

Qualités technologiques des grains: Le rendement à l'usinage des variétés améliorées comme locale varie de 60 à $65 \%$ mais les résultats restent statistiquement homogènes telle que présenter dans le tableau 4. Le poids de mille grains (PMG) diffère entre les variétés $(p<0,05)$ et varie de 16,92 (Kitombe) à $28,44 \mathrm{~g}$ (Lienge). Etant donné qu'il est déjà établi une corrélation positive entre le PMG et le rendement de riz en grains, la variété Lienge serait probablement la plus productive entre ces cinq variétés si les autres paramètres liés au rendement évoluent dans le même sens (Liboga et al., 2018). II est connu que le rendement à l'usinage est un caractère variétal (Faur et Mazaud, 1995) mais plusieurs facteurs peuvent lui influencé notamment le milieu de culture, la teneur en eau au moment de l'usinage, le type de machine utilisée pour l'usinage, le degré de maturité au champ (Liboga et al., 2018). Le pourcentage des brisures par contre a montré une variabilité entre les variétés testées $(p<0,05)$. La variété Kitombe dont les grains sont moins longs a produit un taux de brisures faible $(3,12 \%)$ comparativement aux autres. Le même constat a déjà été fait par d'autres auteurs qui ont abouti aux conclusions selon lesquelles, le pourcentage de brisures bien que aussi influencé par l'environnement, le mode d'usinage, la concentration de $\mathrm{CO}_{2}$ de l'air lors de formation de grains (Usui et al., 2015) mais reste fortement corrélé avec la taille des grains (Faur et Mazaud, 1995). Les grains de tailles courtes et de grosse épaisseur produisent souvent un taux de brisures faible comparativement aux grains de taille longue et effilés (Laignelet et Marie, 1983). Cependant, beaucoup d'autres dommages d'usinage des grains proviennent des grains fissurés qui causent la rupture du riz pendant la mouture (Sotoshi et al., 2016). Ces fissures sont induites par le stress physique accumulé dans l'endosperme par le remplissage inégal résultant de l'absorption inégale d'eau. Récemment, il a été démontré que les hautes températures plus particulièrement au début de la maturation (Takahashi et al., 2002), en particulier le jour (Nagata et al., 2004), sont des facteurs déterminant de la formation de fissures des grains. C'est ainsi que l'irrigation continue avec de l'eau courante réduit le risque de formation de fissures de grains en riziculture irriguée. L'Union Européenne a établi en 1998 d'après Faur et Mazaud (1995) une classification de riz blanchi basée sur le pourcentage de brisures, de grains endommagés et de grains crayeux. Riz avec maximum $5 \%$ de brisures ( $15 \%$ pour le riz précuit) et pas plus de $1 \%$ de grains endommagés et crayeux est de qualité supérieure. Riz avec maximum $10 \%$ de brisures (25\% pour le riz précuit) est de qualité moyenne; riz avec jusqu'à $50 \%$ de brisures est un riz de second choix. Pour cette catégorie, il est de l'obligation de mentionner sur l'emballage le pourcentage de brisures. Pour ces deux dernières catégories, la proportion de grains endommagés et crayeux ne doit pas être plus de 6\%.

Composition en éléments chimiques: La teneur approximative en Fer, en Magnésium et en Calcium a été étudiée et est montrée par le tableau 4, elle varie de 0,39 (Lienge) à $0,71 \%$ (Kitombe) pour le Magnésium, de 0,53 à 0,71\% pour le Fer et de 0,05 à $0,072 \%$ pour le Calcium. Cependant, hormis la teneur 
en Magnésium où il s'est observé une différence significative $(p<0,05)$, aucune différence n'a été observée pour la teneur en Fer et pour la celle en Calcium. Les résultats de notre étude se rapprochent des moyennes déjà établies pour le riz (Oryza sativa L.) de façon générale par Adair (1972), Favier (1989) et par Mihidjay (SD).

Propriétés de riz cuit : Le comportement à la cuisson a été étudié et est présenté dans le tableau 4. II existe de différences significatives entre variétés $(p<0,05)$ pour le volume global de gonflement (VGG) et pour le pouvoir d'élongation ( $E \%$ ), qui varie de $36,74 \%$ (Nerica7) à 50,63\% (Kitombe). Dans ce tableau, il est clairement montré que la variété Kitombe a un pouvoir de gonflement plus élevé que les autres variétés ce qui corrobore ainsi des informations (non publiées) selon lesquelles le pouvoir de gonflement de cette variété est l'un des points clé qui justifie sa préférence par les consommateurs. Cette différence est attribuable à la quantité d'amylose que contient chaque variété (Singh et al., 2005).

Tableau 4: qualité technologiques des grains de riz et teneur en éléments chimiques

\begin{tabular}{|c|c|c|c|c|c|c|c|c|}
\hline \multirow[b]{2}{*}{ Variété } & \multicolumn{3}{|c|}{ Qualité technologique } & \multicolumn{3}{|c|}{$\begin{array}{l}\text { Teneur en éléments } \\
\text { chimiques }\end{array}$} & \multicolumn{2}{|c|}{$\begin{array}{l}\text { Comportement à la } \\
\text { cuisson }\end{array}$} \\
\hline & $\begin{array}{l}\text { Rendement à } \\
\text { l'usinage (\%) }\end{array}$ & \begin{tabular}{|l}
$(\%)$ de \\
brisures
\end{tabular} & PMG (g) & $\mathrm{Ca}(\%)$ & $\operatorname{Mg}(\%)$ & $\begin{array}{c}\mathrm{Fe} \\
(\%)\end{array}$ & VGG $(\mathrm{Cm})$ & $\begin{array}{r}E \\
(\%)\end{array}$ \\
\hline Lioto & $62,17 a$ & $4,57 a$ & $21,6 \mathrm{~b}$ & $0,05 a$ & 0,58 & $0,58 a b$ & $4,87 \mathrm{~b}$ & $43,13 b$ \\
\hline Liboga & $61,21 a$ & $4,31 a$ & $22,55 a b$ & $0,07 a$ & 0,47 & $0,71 \mathrm{a}$ & $4,09 c$ & $35,18 \mathrm{c}$ \\
\hline Lienge & $60,70 a$ & $4,59 a$ & $28,45 a$ & $0,07 a$ & 0,39 & $0,60 a b$ & $4,40 \mathrm{c}$ & $41,38 b$ \\
\hline Nerica7 & $61,21 a$ & $4,05 a$ & $24,30 a b$ & $0,07 a$ & 0,50 & $0,62 a b$ & $4,28 \mathrm{c}$ & $36,74 b$ \\
\hline Kitombe & $65,68 a$ & $3,12 b$ & $16,92 b$ & $0,07 a$ & 0,71 & $0,53 b$ & $5,96 a$ & $50,60 a$ \\
\hline MG & 62,19 & 4,12 & 22,70 & 0,06 & 0,61 & 0,53 & 4,72 & 41,40 \\
\hline $\mathrm{S}^{2}$ (variété) & 16,54 & 1,42 & 70,17 & 0,00033 & 0,015 & 0,055 & 1,685 & 111,34 \\
\hline C.V(\%) & 4,61 & 3,88 & 12,9 & 25,75 & 2,11 & 10,00 & 3,170 & 6,83 \\
\hline LSD 0,05 & $6,25^{\mathrm{NS}}$ & 0,90 & 6,32 & $0,03^{\mathrm{NS}}$ & $0.02^{* * *}$ & $0,13^{\mathrm{NS}}$ & $0,34^{* * *}$ & $6,53^{* \star *}$ \\
\hline
\end{tabular}

Légende : Ns: différence non significative pour $p=5 \% ;{ }^{* * *}$ : différence significative pour $p=0,1 \% ;{ }^{* *}$ : différence significative pour $\mathrm{p}=1 \%$

\section{CONCLUSION ET APPLICATION DES RESULTATS}

Cette étude de la qualité physico-chimique et technologique de cinq variétés de riz pluvial a permis les découvertes de plusieurs informations en rapport avec la qualité physico-chimique et technologique des variétés étudiées. Considérant le critère taille plus particulièrement la longueur, on constate que les grains (riz blanchi) des différentes variétés ont une moyenne de longueur supérieure à $6,0 \mathrm{~mm}$ et peuvent en conséquence être considérées bonnes dans les échanges commerciaux conformément à la classification de I'UE, mais ne permettent d'être classées dans la catégorie de riz de qualité supérieure car le pourcentage de brisures dépasse largement le $1 \%$ et contiennent plus de $1 \%$ de grains endommagés

\section{REMERCIEMENTS}

Cette recherche a été supportée par la fondation internationale pour la science (IFS) Stockholm, Suède à et des grains non uniforme dans une même variété (données non montrées). La variété locale dite Kitombe regorge des qualités notamment teneur élevée en protéine, Mg et le pouvoir de gonflement. Ce dernier serait parmi les points forts de cette variété de riz, c'est ainsi que dans le contexte de paurreté que sont confrontés les congolais en générale, les consommateurs de Kisangani, portent leur choix à cette variété pour permettre aux familles les plus démunies de se partager les petites quantités. Les résultats trouvés dans ce travail s'appliquent bien en sélection et amélioration génétique de plantes car ils permettent de faire le choix de géniteurs sur de leurs caractéristiques physico-chimique et technologiques.

travers la bourse accordée à Monsieur Bienvenu Liboga. 


\section{RÉFÉRENCES BIBLIOGRAPHIQUES}

Adair C.R.1972.Production and utilisation of rice. In: D.F. Houston (éd).Rice chemistry and technology. Am.Assoc. of cereal chemists, St. Paul, Minnesota, pp.125-151

AOAC, 1995.Officiel Méthode of Analysis. Association of Agricultural Chemist, Washington D.C. 34p

Béminga B.M.2011.Caractérisation chimique et minérale des grains de mil [Pennisetum glaucum (L.) RBr.] de Cote d'Ivoire. Inter.J.Chem.Sci.5(5):2039-2044.

Centre du riz pour l'Afrique (ADRAO) ,2009.Sélection végétale participative du riz: Manuel du technicien. Cotonou, Benin: Centre du riz pour I'Afrique (ADRAO) 126p.

Delwiche S.R., McKenzie K.S., Webb B.D.1996.Quality characteristics in rice by near-infrared reflectance analyse of whole grain milled samples. Cereal. Chem. 73:257-263.

Dodelmann J.P.1976. Rizicultures pratiques1-Riz irrigué. Techniques vivantes, ACCT, Paris, 220p

Dubois M. Gilles K.M., Hamilton J.K., Rebers P.A., Smith F.1956.Colorimetric method for determination of sugars and related substances. Anal.chim., 28 (3) : 350-356.

Faure J., Mazaud F.1995.La Qualité du riz dans I'union Européenne. Agricult. et dévelop. $n^{\circ} 6$.

Favier J.C.1989.Valeur nutritive et comportement des céréales au cours de leurs transformations. Céréales en régions chaudes AUPELF-UREF, eds John Lebbey Eurotext Paris, 285-297

International rice research institute (IRRI), 2002.Standard evaluation system for rice (SES).Laos Banos Manila, 56p.

Islam M.M., Kayesh E. Zaman E. Haque M.M.2018.Evaluation of Rice (Oryza sativa L.) Genotypes for Drought Tolerance at Germination and Early Seedling Stage. The Agriculturists 16(1):44-54.

Juliano B.0.1971.A siplified assay for miller-rice amylose. Cereal. Sc. Today: 16:334-340.

Juliano B.O., Tuano A., Paul P.2019. Gross structure and composition of the rice grain Philippines. Philippine Rice Research Institute. 2:32-53.

Kim K-M. Kuk Y.I., Kim W., Back K., Guh J.O., Shin MS.2006. Quality of rice lines transformed with protox gene of Bacillus subtilis. Korean, J.Breed. 38(1):44-50.

Laignelet B. et Marie R.1983.Fluctuation de la qualité technologique du riz en fonction du génotype et du milieu, Agronomie, EDP sciences, 3 (2):179-183.

Liboga O.B., Litucha B.J.2018.Evaluation de quelques variétés de riz pluvial (Orysa sativa L.) dans les conditions agro écologiques de Kisangani, R.D.Congo. Annales IFA-Yangambi (2):35-46.

Mateso B., Bantodisa K., Lienge B.2003.Liboga, a new short-duration variety released in République Démocratique du Congo. IRRN 28.1/2003, P37.

Mihidjay(SD).Caractérisation des variétés de riz cultivées à Mohéli, îles de Comores https://www.qualireg.org/content/dowload/1637 8/22479/version/1. Consulté le 20 Janvier 2019.

Monsoor M.A., Proctor A., Siebenmorgen T.J.2004.Surface lipid and FFA content of head and broken rice produced by milling after different drying treatments, Cereal Chem.81:705-709.

Nagata K., Takita T., Yoshinaga S., Terashima K., Fukuda A.2004.Effect of air temperature during the early grain-filling stage on grain fissuring in rice. Japanese J. of Crop. Sc., 73:336-342.

Okpala NE., Duan L., Shen G, Zhang G, Qi X. 2017.Comparisons of Cooking and Eating Qualities of Two Indica Rice Cultivars. J.Rice Res 5:180.doi:10.4172/2375-4338.1000180.

Pandey V. et Shukla A.2015.Acclimation and Tolerance Strategies of Rice under Drought Stress. Rice Sc. 22(4):147-161. http://dx.doi.org/10.1016/j.rsci.2015.04.001.

Satoshi M., Hiroshi W., et Yuji M.2016.Countermeasures for heat damage in rice grain quality underclimatechange.PlantProd.Sc19:(1)1-11. doi:10.1080/1343943x.2015.1128114.

Simonelli C., Abbiati A., Cormegna M.2016.Physicochemical Characterization of some Italian rice varieties. La Rivista di scienza deLL'aLimentazione, numeRo 1 , gennaio-apRiLe, anno 45.

Singh N., Kaur L., Sodhi N.S., Sekhon K.S.2005.Physicochemical, cooking and textural properties of milled rice from different Indian rice cultivars, Food Chemistry, 89: 253259.

Takahashi W., Ojima T., Nomura M., Nabeshima M.2002.Development of a model for predicting 
cracked rice kernel of rice cultivar "Koshihikari". The Hokuriku Crop Sci., 37:4851.

Tomonori Y., Kazuki S.2016.Factors affecting farmers' adoption of NERICA upland rice varieties: the case of a seed producing village in central Benin. Food Sc. 8:197-209.

Usui Y., Sakai H., Tokida., Nakagawa H., Hasegawa, T.2015.Rice grain yield and quality responses to free-air $\mathrm{CO}_{2}$ enrichment combined with soil and water warming. Glob.Chang.Biol. 22(3): 